\title{
Comparative Effectiveness of Treatments for Binge-Eating Disorder: Systematic Review and Network Meta-Analysis
}

\author{
Christine M. Peat ${ }^{1,2 *}$ (D), Nancy D. Berkman ${ }^{3}$, Kathleen N. Lohr ${ }^{3}$, Kimberly A. Brownley $^{1}$, Carla M. Bann ${ }^{3}$, \\ Katherine Cullen ${ }^{3}$, Mary J. Quattlebaum ${ }^{1}$ \& Cynthia M. Bulik ${ }^{1,4,5}$ \\ ${ }^{1}$ Department of Psychiatry, University of North Carolina, Chapel Hill, NC, USA \\ ${ }^{2}$ Department of Neurosurgery, University of North Carolina, Chapel Hill, NC, USA \\ ${ }^{3}$ RTI International, Research Triangle Park, NC, USA \\ ${ }^{4}$ Department of Nutrition, University of North Carolina, Chapel Hill, NC, USA \\ ${ }^{5}$ Department of Medical Epidemiology and Biostatistics, Karolinska Institutet, Stockholm, Sweden
}

\begin{abstract}
Psychological and pharmacological interventions for binge-eating disorder have previously demonstrated efficacy (compared with placebo or waitlist control); thus, we aimed to expand that literature with a review of comparative effectiveness. We searched MEDLINE, ${ }^{\circledR}$ EMBASE, ${ }^{\circledR}$ Cochrane Library, Academic OneFile, CINAHL ${ }^{\circledR}$ for binge-eating disorder treatment articles and selected studies using predetermined inclusion and exclusion criteria. Data were sufficient for network meta-analysis comparing two pharmacological interventions; psychological interventions were analysed qualitatively. In all, 28 treatment comparisons were included in this review: one pharmacological comparison (second-generation antidepressants versus lisdexamfetamine) and 26 psychological comparisons. Only three statistically significant differences emerged: lisdexamfetamine was better at increasing binge abstinence than second-generation antidepressants; therapist-led cognitive behavioural therapy was better at reducing binge-eating frequency than behavioural weight loss, but behavioural weight loss was better at reducing weight. The majority of other treatment comparisons revealed few significant differences between groups. Thus, patients and clinicians can choose from several effective treatment options.

Received 12 December 2016; Revised 19 March 2017; Accepted 20 March 2017
\end{abstract}

Keywords

binge-eating disorder; comparative effectiveness; systematic review; treatment

*Correspondence

Christine M. Peat, PhD, Department of Psychiatry, University of North Carolina at Chapel Hill, CB \#7160, Chapel Hill, NC 27599-7160, USA. Tel: (984) 974-3804; Fax: (984) 974-3780.

Email: christine_peat@med.unc.edu

Published online 3 May 2017 in Wiley Online Library (wileyonlinelibrary.com) DOI: 10.1002/erv.2517

\section{Introduction}

Binge-eating disorder (BED)—characterized by consuming large amounts of food with an associated sense of loss of controlmerits particular attention by clinicians and patients alike given its elevation to an independent diagnosis in the most recent version of the Diagnostic and Statistical Manual (DSM-5) (American Psychiatric Association, 2013). BED is common among adults in the United States; lifetime prevalence is estimated at $2.8 \%$ (Hudson, Hiripi, Pope, \& Kessler, 2007). Prevalence is higher among obese individuals, particularly those seeking treatment for weight loss (Bruce \& Wilfley, 1996; Grucza, Przybeck, \& Cloninger, 2007; Spitzer et al., 1993a; Spitzer et al., $1993 b)$. BED is considered to be a public health problem because of its impact on psychiatric, physical, and social functioning (Hudson et al., 2007; Kessler et al., 2013; Whisman, Dementyeva, Baucom, \& Bulik, 2012).

BED thus warrants a rigorous systematic review; this paper presents the comparative effectiveness results of treatments for individuals with this condition. It reports on and updates findings from a larger systematic review on the management and outcomes of BED recently completed for the US Agency for Healthcare Research and Quality by the RTI-University of North Carolina Evidence-based Practice Center (Berkman et al., 2015); that systematic review had updated our 2006 review on BED and related eating disorders (Berkman et al., 2006; Brownley, Berkman, Sedway, Lohr, \& Bulik, 2007). Findings from efficacy studies about the benefits and harms of psychological and pharmacologic therapies for adults with BED were published in June 2016 (Brownley et al., 2016). Cognitive behavioural therapy (CBT), second-generation antidepressants (SGAs), lisdexamfetamine, and topiramate all reduced binge eating and related psychopathology; the latter two medications also produced reductions in weight. Thus, each of these interventions offered benefits. Some side effects were associated with pharmaceutical interventions (such as those from lisdexamfetamine for gastrointestinal upset or headaches, from topiramate for sympathetic nervous system 
arousal, and from SGAs for sleep disturbance); no adverse effects were reported for psychological treatments although they were not universally assessed (Brownley et al., 2016).

As a complement to efficacy studies, comparative effectiveness reviews are designed to help inform health care decisions by comparing outcomes, drawn ideally from head-to-head comparative trials, from among treatments that have already demonstrated efficacy (i.e., those with active comparators rather than placebo, waitlist, or usual care). Such reviews help inform researchers, patients, and clinicians alike on which of the available efficacious interventions might work best for the given population or illness. Comparative effectiveness reviews contribute to the evidence base necessary to make decisions about optimal treatments (i.e., increasing benefits and minimizing harms). A comparative effectiveness review for BED is thus both timely and appropriate given that the efficacy of several interventions has been established (Berkman et al., 2015; Brownley et al., 2016), but that relatively little is understood regarding which interventions for BED may be more effective and for whom.

Several approaches for treating BED patients have been evaluated in the literature; these include both pharmacological and psychological interventions (Berkman et al., 2015; Brownley et al., 2016; Brownley et al., 2007; Peat, Brownley, Berkman, \& Bulik, 2012). With regard to pharmacological

interventions, studies have evaluated the efficacy of various antidepressants, appetite suppressants, and anticonvulsant medications. In 2015, lisdexamfetamine (a central nervous system stimulant originally marketed as a drug for attention deficit hyperactivity disorder) became the first medication that the US Food and Drug Administration (FDA) approved for treating BED patients (U.S. Food and Drug Administration, 2015). For psychological interventions, the majority of studies have involved CBT, interpersonal psychotherapy (IPT), and behavioural weight loss (BWL).

Within these larger categories of psychological interventions, investigators have developed distinct variations for delivering the treatments (e.g., therapist-led and self-help; individual and group format; different treatment durations). Researchers developed the variations to try to answer important empirical questions that would deconstruct the essential components of treatment (e.g., scalability and level of necessary therapist involvement). This practice, however, has fragmented the available evidence on the psychological management of BED. The result is largely a body of literature of 'one-off' studies on psychological management that is difficult to evaluate collectively. Policymakers, however, require actionable evidence on differences across treatment modalities, particularly in light of an anticipated increased focus on BED in clinical care, corresponding to its recent DSM-5 designation as a distinct eating disorder, and concern about the paucity of BED psychological treatment expertise available generally. Thus, efforts to synthesize the literature are crucial at this time.

Complicating this challenge are the lack of replication trials across both pharmacological and psychological interventions and a paucity of well-designed studies that directly compare the core psychological treatments and their variants (e.g., therapistled CBT versus self-help CBT). Together, these limitations hinder the field's ability to draw meaningful conclusions about which treatments are most beneficial for individuals with BED. Being able to draw defensible conclusions has become more critical with BED now an independent disorder in the DSM-5, the increasing awareness about the condition, and the predicted increase in treatment seeking (Marek, Ben-Porath, Ashton, \& Heinberg, 2014; Trace et al., 2012).

We present here the specific results from our larger systematic review about the comparative effectiveness of selected treatments for adults with BED. Of particular importance are four major sets of outcomes: binge-eating outcomes (e.g., abstinence and bingeeating frequency), eating-related psychopathology outcomes (e.g., obsessions and compulsions), weight-related outcomes [e. g., body mass index (BMI)], and general psychological outcomes (e.g., depressive symptoms). Other outcomes, such as quality-oflife benefits and risks of adverse events, are of interest as well. We also discuss the clinical and scientific implications of our findings and highlight important directions for future research and clinical practice.

\section{Methods}

\section{Eligibility criteria}

Eligible studies for these specific analyses were randomized controlled trials (RCTs) with sample sizes of 10 or more and published in English. Detailed information on inclusion and exclusion criteria is available in the full report (Berkman et al., 2015). Using the population, intervention, comparators, outcomes, timing, and settings (PICOTS) framework, our principal inclusion criteria pertaining to this paper included the following:

- Individuals of all races, ethnicities, and cultural groups who meet DSM-IV or DSM-5 criteria for BED;

- Psychological, behavioural, pharmacological, or complementary and alternative treatments or combinations of treatments;

- Two or more active comparators (from PICOTS criteria);

- Final health outcomes or intermediate health outcomes, such as biomarkers, that can be linked directly to final physical health outcomes;

- Outcomes evaluated at the end of treatment or later followup (or both); and

- No limits on settings.

\section{Data sources and search strategy}

An experienced research librarian searched MEDLINE, ${ }^{\circledR}$ EMBASE, ${ }^{\circledR}$ Cochrane Library, Academic OneFile, and the Cumulative Index to Nursing and Allied Health Literature (CINAHL ${ }^{\circledR}$ ) using a predefined list of search terms and medical subject headings for articles indexed through 17 November 2015. The same librarian updated the MEDLINE ${ }^{\circledR}$ search to find any relevant articles indexed through 12 May 2016. Appendix A presents the full search strategy. We searched for unpublished and grey literature from trial registries (e.g., ClinicalTrials.gov). For older studies on BED, we searched the relevant portion of the reference list of our 2006 review, Management of Eating Disorders; however, we did not rely on the earlier review to 
identify studies (Berkman et al., 2006; Berkman, Lohr, \& Bulik, 2007; Brownley et al., 2007). We also hand searched reference lists and relevant systematic reviews and reviewed articles suggested by members of our technical expert panel.

\section{Study selection}

Trained pairs of research team members selected abstracts for full-text review if they met the predefined eligibility inclusion and exclusion criteria. We conducted a dual review of all trials selected for full-text review. We excluded studies at this stage if both reviewers agreed that it did not meet eligibility criteria. If reviewers did not agree on inclusion, they resolved the disagreement through discussion or with help of a third, senior reviewer.

\section{Data abstraction}

Based on the PICOTS framework for the full review (Berkman et al., 2015), we abstracted information on trial characteristics, study designs, methods, and results. One member of the research team abstracted relevant data from each included article. A senior member of the research team reviewed each abstraction for accuracy and completeness.

\section{Risk of bias assessment and strength of evidence grading}

We assessed the risk of bias for all included RCTs using the Cochrane risk-of-bias tool (Higgins et al., 2011). Two independent reviewers assessed the potential for selection bias, performance bias, attrition bias, detection bias, and outcome reporting bias and gave each study a grade of low, medium, or high risk of bias (Berkman et al., 2015). Disagreements on riskof-bias ratings were regularly resolved through discussion by the two reviewers or consultation with a third team member on an as-needed basis.

We graded the strength of evidence (SOE) for key outcomes of treatment comparisons as high, moderate, low, or insufficient, based on the Evidence-based Practice Center Methods Guide

(Berkman et al., 2013; Berkman et al., 2014). The five domains, assessed independently by two reviewers, include study limitations, consistency, directness, precision, and reporting bias. The full report documents the risk-of-bias and SOE methods and grades (Berkman et al., 2015).

\section{Data synthesis of pharmaceutical interventions}

Only pharmacological interventions met the minimum threshold for pooled analysis (e.g., three or more studies with reasonably homogenous interventions, populations, and outcomes). Because these trials (of SGAs and lisdexamfetamine) were efficacy trials (i.e., placebo-controlled), we were interested in determining whether their effects differed. To explore this issue, comparative effectiveness analyses would ideally be drawn from head-to-head trials that directly compare two active interventions. Our search of the published literature revealed only a single head-to-head trial of pharmacological interventions (Leombruni et al., 2008). Therefore, as discussed in the succeeding text we used network meta-analysis to compare outcomes of lisdexamfetamine therapy with those of the SGAs (as a class) at the end of treatment. Network meta-analysis allows for the estimation of comparative treatment effects in the absence of head-to-head data that are frequently unavailable. Thus, researchers can pool results from separate trials involving the same population, comparison (e.g., placebo or wait-list), and outcomes to better visualize the broader picture of evidence and help identify which treatments might be more effective.

We conducted network meta-analyses using a graph-based frequentist approach described by Rücker (2012) and implemented in the NETMETA package in R (https://cran.r-project. org/web/packages/netmeta/index.html) (Neupane, Richer, Bonner, Kibret, \& Beyene, 2014). In addition, as a sensitivity check, we fit the models using a Bayesian approach (van Valkenhoef et al., 2012) implemented in the GEMTC package in R (https://cran.rproject.org/web/packages/gemtc/gemtc.pdf) (van Valkenhoef \& Kuiper, 2016); it produced similar results (not shown). Results from network (also known as indirect) meta-analyses tend to agree with head-to-head trials when component studies are similar and treatment effects are expected to be consistent in patients in different trials (Glenny et al., 2005). To conduct network metaanalyses, we included all the placebo-controlled pharmaceutical trials that were homogenous in study populations and outcome assessments. To account for potential between-study heterogeneity, we estimated the indirect treatment effects based on random effects models. We estimated relative risks and 95\% confidence intervals (CIs) as the effect measures for categorical outcomes and mean differences (and 95\% CIs) for continuous outcomes [binge-eating days and scores on the Yale Brown Obsessive Compulsive Scale Modified for Binge Eating (YBOCSBE)] (Deal, Wirth, Gasior, Herman, \& McElroy, 2015).

\section{Data synthesis of psychological interventions}

Although our search did reveal head-to-head comparisons of psychological interventions, the psychological interventions trials did not provide sufficiently similar data to meet the minimum threshold of three similar studies for pooled analysis. For that reason, we analysed these data only qualitatively.

Qualitative data synthesis was a collaborative process among senior reviewers based on an existing protocol; we based our groupings of studies and subsequent findings on judgments about the similarity of interventions and outcomes measured and the homogeneity of patient populations. For example, in our judgment therapeutic interventions involving individual-based versus group-based formats in CBT trials were ones that we considered sufficiently dissimilar to conceivably affect outcomes. In these situations, we did not combine information across therapy modalities, but we do present information about these unique studies in results in the succeeding text.

We were not able to comment on trials comparing pharmacological interventions with psychological interventions. Our rigorous search did not reveal any trials that directly compared a single pharmacological intervention with a single behavioural intervention thereby preventing any qualitative analysis. We were also unable to conduct network meta-analysis given that the comparators in the available efficacy trials differed. In the pharmacological efficacy trials, placebo was used as the comparator against the active intervention, whereas in psychological efficacy trials, the comparator used was waitlist control. Conceptually, placebo and waitlist comparators are 
dissimilar as one involves active concurrent participation (receiving the placebo; Gupta \& Verma, 2013); waitlist implies no concurrent participation. Participants randomized to a placebo condition are blinded to whether or not they are receiving the active intervention. Thus, expectations about treatment outcome are relevant in ways that are not as salient in waitlist control groups where participants are fully aware they are not actively receiving an intervention. Also, the waitlist control condition is designed such that participants do not have any contact with study personnel after the first (baseline) assessment, until the intervention period has ended. In contrast, participants in a placebo-controlled trial may have continued and consistent contact with study personnel by way of visits for treatment administration, phone contact to schedule visits, on-going measurement of interim outcomes and general attention by study personnel. These non-specific factors have been found to account for much of the outcome variance in clinical trials (Chatoor \& Krupnick, 2001): thus, control conditions with varying levels of non-specific factors should be considered conceptually distinct.

Our search did not reveal comparative effectiveness trials that involved combination approaches (i.e., combining a pharmacological and psychological intervention) versus a different active treatment. All combination approaches were placebo-controlled trials and therefore covered in the previously published efficacy results (Brownley et al., 2016) and in the full report (Berkman et al., 2015).

\section{Results}

\section{Description of studies}

Our searches identified 4,794 potentially relevant citations (Figure 1). The full systematic review included 87 of these publications; this paper focuses on the 42 studies providing evidence on the comparative effectiveness of treatment among adults with BED. Consistent with our prespecified process, for 11 of the 30 included studies, the two independent risk-of-bias assessors needed to reconcile their final rating. In all cases, the initial risk-of-bias rating was either low or medium. Seven were finalized as medium risk-of-bias, whereas four were finalized as low.

A total of 12 trials provided evidence of comparative effectiveness of pharmacological interventions. A single head-tohead trial (Leombruni et al., 2008) compared two SGAs: fluoxetine and sertraline. The remaining 11 pharmacological trials (reported in 12 articles) contributed to the network meta-analysis of major outcomes (trial characteristics reported in Appendix B). Of these, eight involved SGAs (Arnold et al., 2002; Grilo, Masheb, \& Wilson, 2005; Guerdjikova et al., 2008; Guerdjikova et al., 2012; Hudson et al., 1998; McElroy et al., 2000; McElroy et al., 2003;

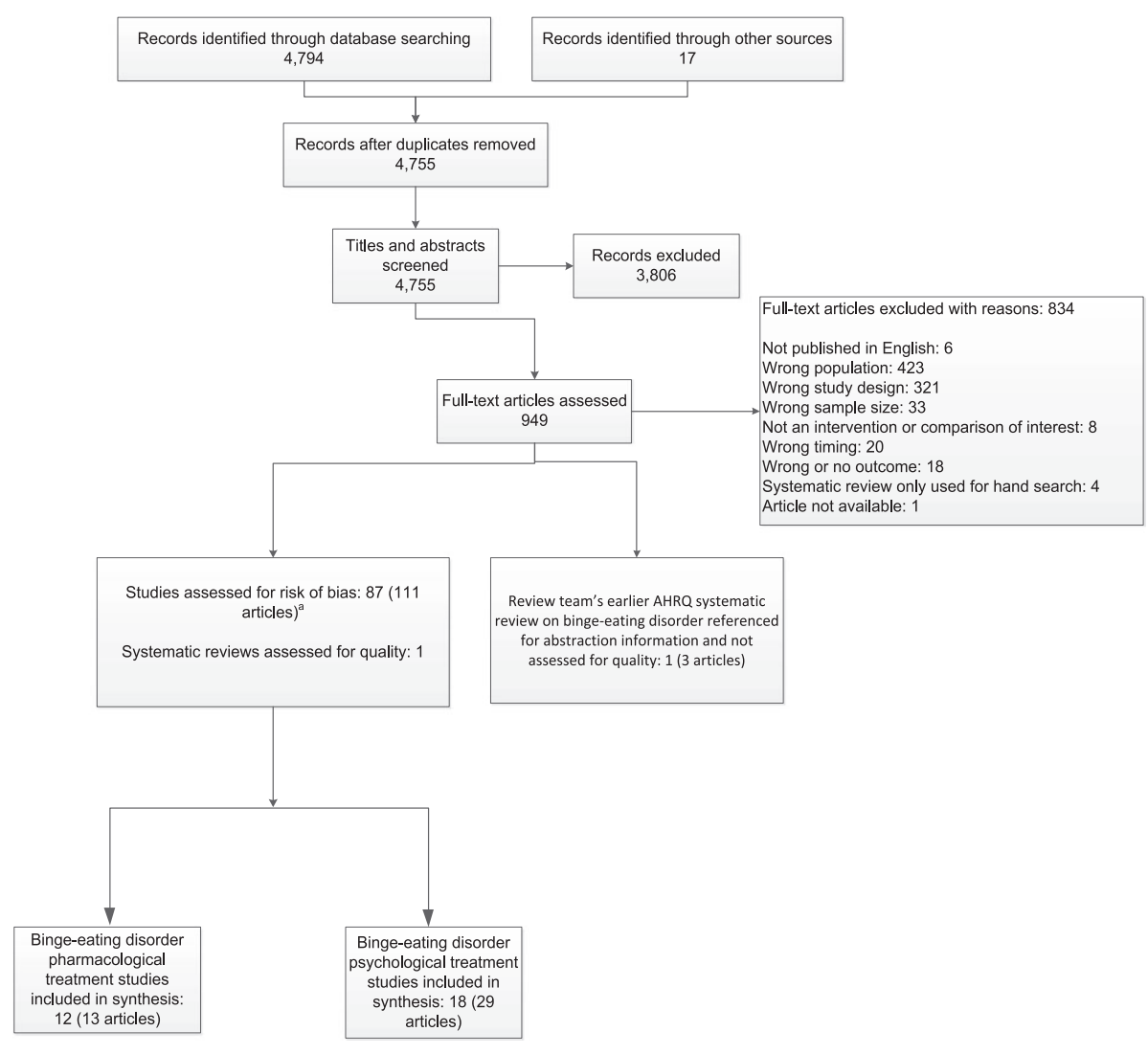

Figure 1. Flow diagram of the literature search process for studies of binge-eating disorder. AHRQ Agency for Healthcare Research and Quality; KQ, key question 
White \& Grilo, 2013) and three involved lisdexamfetamine (McElroy et al., 2015a; McElroy et al., 2015b; Shire Development LLC, 2014a, 2014b).

The evidence of comparative effectiveness of psychological treatments was reported in 18 trials (in 29 articles) (Carter \& Fairburn, 1998; Castelnuovo, Manzoni, Villa, Cesa, \& Molinari, 2011; Cesa et al., 2013; De Zwaan et al., 2005; Grilo \& Masheb, 2005; Grilo, Masheb, Wilson, Gueorguieva, \& White, 2011; Grilo, White, Wilson, Gueorguieva, \& Masheb, 2012; Hilbert et al., 2012; Hilbert, Hildebrandt, Agras, Wilfley, \& Wilson, 2015; Hilbert \& Tuschen-Caffier, 2004; Le Grange, Gorin, Dymek, \& Stone, 2002; Masheb \& Grilo, 2005; Masheb, Grilo, \& Rolls, 2011; Munsch et al., 2007; Munsch, Meyer, \& Biedert, 2012; Peterson, Mitchell, Crow, Crosby, \& Wonderlich, 2009; Peterson et al., 2001; Peterson et al., 1998; Pisetsky et al., 2015; Ricca et al., 2010; Riva, Bacchetta, Baruffi, \& Molinari, 2002; Robinson \& Safer, 2012; Safer \& Joyce, 2011; Safer, Robinson, \& Jo, 2010; Sysko, Hildebrandt, Wilson, Wilfley, \& Agras, 2010; Tasca, Balfour, Presniak, \& Bissada, 2012; Tasca et al., 2006; Wilfley et al., 2002; Wilson, Wilfley, Agras, \& Bryson, 2010). These 18 trials examined various forms of CBT, IPT, BWL, dietary approaches, and inpatient interventions for managing BED (trial characteristics reported in Appendices C-F). The 18 trials presented 26 treatment comparisons. Of these, four were replicated in more than one trial and 22 were confined to a single trial each.

\section{Comparative effectiveness of second-generation antidepressants versus Lisdexamfetamine}

As described in Appendix B, 11 trials were available for network meta-analysis comparing SGAs (as a class) with lisdexamfetamine at the end of treatment (Arnold et al., 2002; Grilo et al., 2005; Guerdjikova et al., 2008; Guerdjikova et al., 2012; Hudson et al., 1998; McElroy et al., 2000; McElroy et al., 2015a; McElroy et al., 2003; McElroy et al., 2015b; Shire Development LLC, 2014a, 2014b; White \& Grilo, 2013). For these analyses, we included information from three trials comparing lisdexamfetamine with placebo and eight trials comparing SGAs with placebo; the SGAs included bupropion, citalopram, duloxetine, escitalopram, fluoxetine, fluvoxamine, and sertraline. A total of 725 participants (517 lisdexamfetamine and 208 SGAs) constituted the treatment arms across these trials. All 11 trials provided data sufficient for the analysis of binge abstinence; six had data on binge-eating frequency (expressed in binge days), and six provided data on binge-eating related obsessions and compulsions [measured on the YBOCS-BE (Deal et al., 2015) total score ranging from 0 to 40].

Figure 2 reports on the meta-analysis results comparing SGAs and lisdexamfetamine for abstinence, binge-eating frequency, and binge-eating obsessions and compulsions. Lisdexamfetamine was associated with statistically significant greater abstinence than SGA, relative risk 1.56 (95\% CI, 1.06 to 2.29; moderate SOE for greater benefit). Differences between SGAs and lisdexamfetamine were not statistically significant for either binge frequency (mean difference in days per week -0.48 ; $95 \% \mathrm{CI},-1.17$ to 0.20 ; low $\mathrm{SOE}$ for no difference) or obsessions and compulsions (mean difference -2.68 ; $95 \% \mathrm{CI},-5.41$ to 0.06 ; low SOE for no difference).

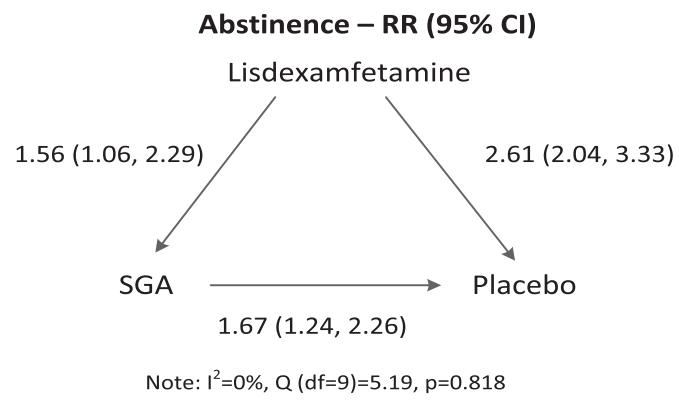

Binge Days Per Week - Mean difference $(95 \% \mathrm{CI})$

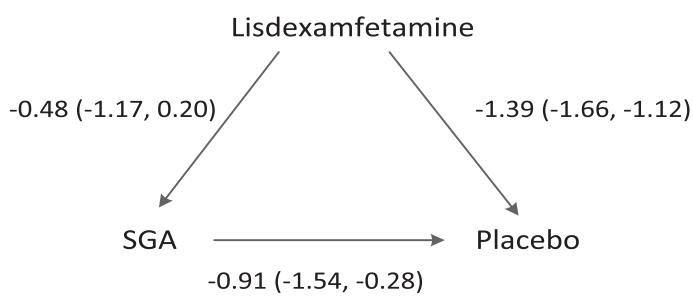

Note: $I^{2}=98.3 \%, Q(d f=4)=236.87, p<0.001$

YBOCS Total Score - Mean difference (95\% Cl)

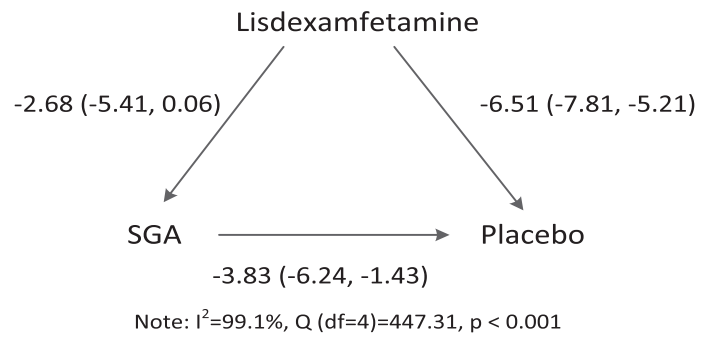

Figure 2. Results of network meta-analyses comparing second generation antidepressants and lisdexamfetamine. $\mathrm{Cl}$, confidence interval; RR, Relative Risk; SGA, second-generation antidepressants; YBOCS, Yale-Brown Obsessive Compulsive Scale Modified for Binge Eating

In short, both SGAs and lisdexamfetamine improved bingeeating outcomes and eating-related psychopathology when compared with placebo (Brownley et al., 2016). Network metaanalysis revealed that lisdexamfetamine was superior to SGAs only in achieving binge abstinence.

\section{Comparative effectiveness of different levels of therapist involvement in delivering cognitive behavioural therapy}

Two trials by the same group of researchers examined different levels of therapist involvement in delivering group-based CBT and reported up to 12-month follow-up data (Peterson et al., 2009; Peterson et al., 2001; Peterson et al., 1998). One was a small trial of 50 patients; the other was a larger trial of 190 patients. Each trial compared (i) therapist-led CBT, (ii) partially therapist-led CBT, and (iii) structured self-help CBT. Although all forms of treatment involved manualized CBT delivered in a 
group setting, the modalities differed in the amount of therapist contact that participants received. In the therapist-led arm, a clinician led the group for the entirety of each session. In the partially therapist-led group, participants watched a pre-recorded psychoeducational video (of the same therapist) during the first half of the session, and the therapist led the group discussion during the second half. In the structured self-help group, participants watched the same pre-recorded psychoeducational video as in the partially therapist-led condition, but a group member facilitated the second half of the session (so the only 'therapist contact' that participants had was through the video). The randomized sample comprised a total of 240 participants (76 therapist-led CBT, 82 partially-therapist led CBT, and 82 structured self-help CBT). Both trials reported on binge abstinence, binge-eating frequency, BMI, and depressive symptoms.

As documented in Table 1, across all comparisons, only two statistically significant differences emerged. Both were from the larger trial $(N=190)$ (Peterson et al., 2009). Specifically, therapist-led CBT was associated with significantly greater binge abstinence and greater reductions in binge-eating frequency than structured self-help CBT at end of treatment; however, differences were not significant at 12-month followup (Peterson et al., 2009). In contrast, the smaller trial did not find significant differences between treatment arms. Because of the inconsistency in the results across studies, SOE for therapist-led CBT versus structured self-help CBT for bingeeating outcomes was insufficient.
Both trials reported improvements in other relevant outcomes (e.g., eating-related psychopathology, BMI, and depressive symptoms). These treatment comparisons, based on level of therapist involvement, were not significantly different at either end of treatment or 12-month followup; we graded SOE as low for no treatment difference for all these other outcomes.

In short, each of the CBT treatment modalities (e.g., therapistled and structured self-help) produced some clinically meaningful changes in abstinence and binge-eating frequency. However, the range of benefits was wide at end of treatment: abstinence ranged from $17.9 \%$ to $86.7 \%$ and binge-eating frequency from an average of 6.3 episodes to 0.4 episodes in the past 28 days (Table 1). A similarly wide range of benefits was also observed in both trials at 12-month followup (Table 1). Thus, the available evidence prevents us from reaching a definitive conclusion about their comparative benefits for treating BED patients.

\section{Comparative effectiveness of therapist-led cognitive behavioural therapy versus therapist-led behavioural weight loss}

Two RCTs compared therapist-led CBT with therapist-led BWL, both delivered in a group format (Grilo et al., 2011; Munsch et al., 2007). Follow-up data were collected for up to 72 months, but the majority of these data were not reported. Although the two trials based CBT on the same manual (Fairburn, 1995; Fairburn, Marcus, \& Wilson, 1993), BWL was based on two different manuals. The Munsch trial (Munsch et al., 2007) used the manual 'Weight Loss with Xenical' (Margraf, 2000), whereas

Table 1 Outcomes of trials comparing variants of cognitive behavioural therapy for binge-eating disorder

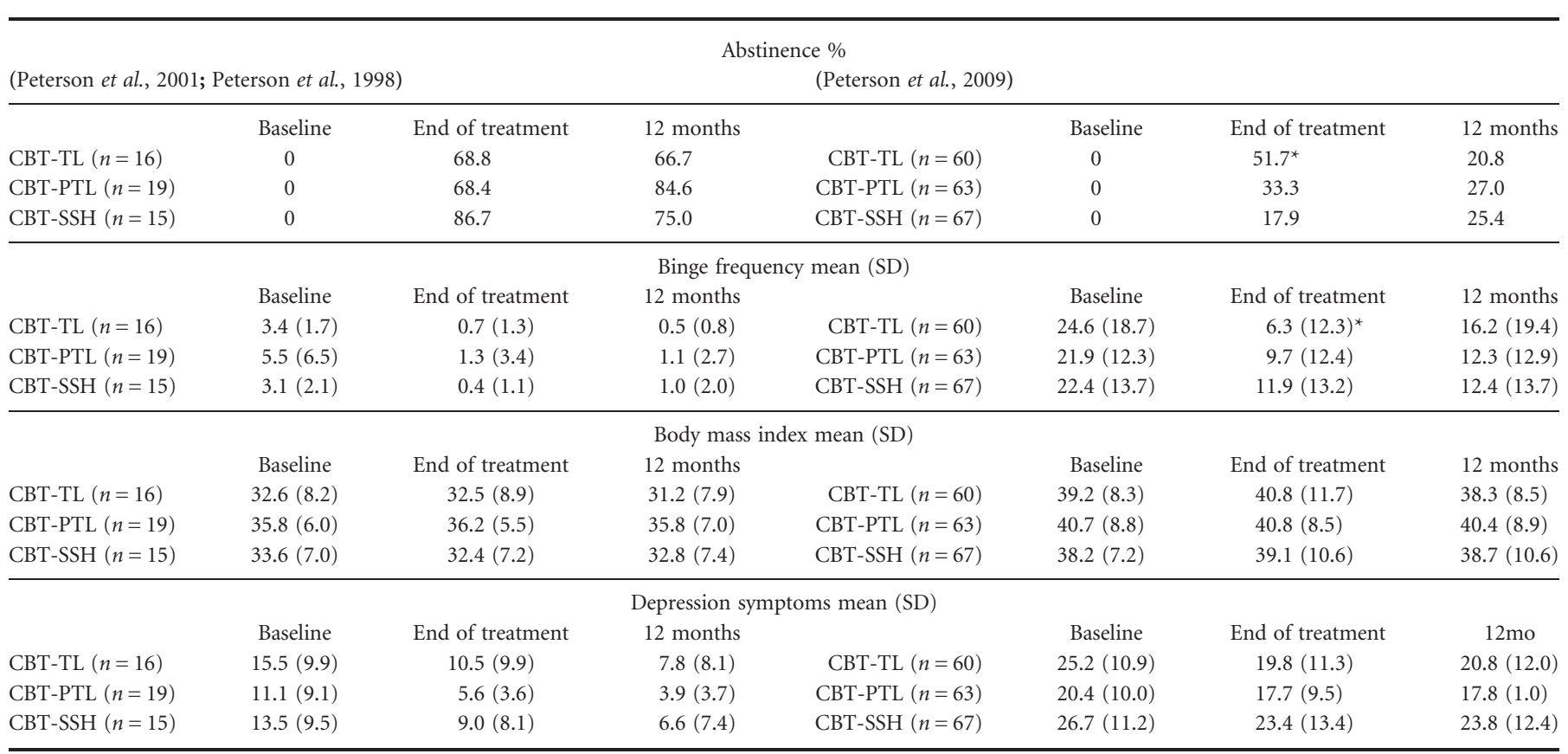

${ }^{*} p<.008$ versus CBT-SSH; all other comparisons were non-significant.

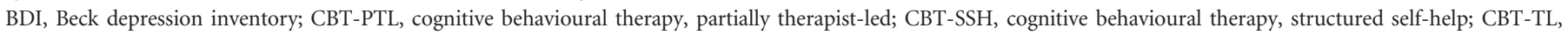
cognitive behavioural therapy, therapist-led; IDS-SR, inventory of depressive symptomatology, self-rated; $n$, number of participants. 
the Grilo trial (Grilo et al., 2011) used the 'Lifestyles, Exercises, Attitudes, Relationships, and Nutrition (LEARN)' manual (Brownell, 2000). The randomized sample comprised 170 participants (89 CBT and $81 \mathrm{BWL}$ ).

As shown in Table 2, both trials reported significantly greater reductions in binge-eating frequency for participants receiving CBT than for those receiving BWL at end of treatment and up to 72 months followup. We graded the SOE as low for CBT being more beneficial for reducing binge eating. However, neither trial reported a significantly greater benefit of CBT on binge abstinence. In fact, in the Munsch trial (Munsch et al., 2007), the percentage of patients abstinent at the end of treatment was significantly lower in the CBT group (41\%) than the BWL group (58\%); however, by 12 -month followup, this differential essentially disappeared. Because of these mixed abstinence results from these two trials, we graded SOE for abstinence as insufficient.

Both trials reported statistically significant greater reductions in BMI for participants receiving BWL than for those receiving CBT at end of treatment (but not followup); we graded SOE as moderate for BMI benefit from BWL. The magnitude of change in BMI was substantial: at end of treatment, those receiving CBT decreased BMI by only an average of 0.41 points whereas those receiving BWL decreased BMI by an average of 2.2 points. However, by 12-month followup, this difference was no longer statistically significant, as those receiving BWL tended to gain weight. Finally, depression symptoms at end-of-treatment and followup improved for patients in both CBT and BWL groups, but the changes did not differ significantly between the two interventions (low SOE for no difference). As with the trials comparing different CBT variations, despite the fact that comparisons between BWL and CBT were mixed for abstinence and not significantly different for depressive symptoms, both treatments consistently produced better outcomes between baseline to the end of treatment across trials and these findings tended to persist at 12-month followup (Table 2).

\section{Summary of the strength of evidence for comparative effectiveness}

Table 3 presents the SOE grades for the comparative analyses presented previously. The majority of grades for comparisons of psychological interventions were low for no difference; those for

Table 2 Outcomes of trials comparing cognitive behavioural therapy with behavioural weight loss for binge-eating disorder

\begin{tabular}{|c|c|c|c|c|c|c|c|c|c|}
\hline \multicolumn{10}{|c|}{ Abstinence \% } \\
\hline \multicolumn{6}{|c|}{ (Munsch et al., 2007; Munsch et al., 2012) } & \multicolumn{4}{|c|}{ (Grilo et al., 2011; Grilo et al., 2012) } \\
\hline & $\begin{array}{l}\text { Baseline } \\
\text { (\%) }\end{array}$ & $\begin{array}{c}\text { End of } \\
\text { treatment }(\%)\end{array}$ & 12 months (\%) & 72 months & & Baseline (\%) & $\begin{array}{c}\text { End of } \\
\text { treatment }(\%)\end{array}$ & $\begin{array}{l}6 \text { months } \\
(\%)\end{array}$ & 12 months $(\%)$ \\
\hline CBT-TL $(n=44)$ & 0 & 41 & 52 & NR & $\begin{array}{l}\text { CBT-TL } \\
(n=45)\end{array}$ & 0 & 44.40 & 51.1 & 51.10 \\
\hline BWL-TL $(n=36)$ & 0 & 58 & 50 & NR & $\begin{array}{l}\text { BWL-TL } \\
(n=45)\end{array}$ & 0 & 37.80 & 33.3 & 35.60 \\
\hline \multicolumn{10}{|c|}{ Binge frequency mean $(\mathrm{SD})$} \\
\hline & Baseline & $\begin{array}{c}\text { End of } \\
\text { treatment }\end{array}$ & 12 months & 72 months & & Baseline & $\begin{array}{c}\text { End of } \\
\text { treatment }\end{array}$ & 6 months & 12 months \\
\hline CBT-TL $(n=44)$ & $3.81(3.47)$ & $0.14(0.45)^{* *}$ & $0.52(1.59)^{*}$ & $\mathrm{NR}^{*}$ & $\begin{array}{l}\text { CBT-TL } \\
(n=45)\end{array}$ & $15.6(8.0)$ & $2.2(3.8)$ & $2.7(8.5)^{* *}$ & $2.4(8.1) * * * *$ \\
\hline BWL-TL $(n=36)$ & $4.10(3.71)$ & $1.15(1.89)$ & $1.50(2.14)$ & NR & $\begin{array}{l}\text { BWL-TL } \\
(n=45)\end{array}$ & $14.9(8.5)$ & $4.6(11.0)$ & $5.5(7.6)$ & $4.6(6.0)$ \\
\hline \multicolumn{10}{|c|}{ Body mass index mean (SD) } \\
\hline & Baseline & $\begin{array}{l}\text { End of } \\
\text { treatment }\end{array}$ & 12 months & 72 months & & Baseline & $\begin{array}{c}\text { End of } \\
\text { treatment }\end{array}$ & 6 months & 12 months \\
\hline CBT-TL $(n=44)$ & $33.6(4.31)$ & $33.58(4.53)$ & $33.10(5.04)$ & $33.5(3.8)$ & $\begin{array}{l}\text { CBT-TL } \\
(n=45)\end{array}$ & $39.3(6.1)$ & $38.5(5.7)$ & $38.7(5.7)$ & $38.3(6.0)$ \\
\hline BWL-TL $(n=36)$ & $34.36(3.74)$ & $32.29(4.00)^{*}$ & $33.18(4.17)$ & $31.5(5.2)$ & $\begin{array}{l}\text { BWL-TL } \\
(n=45)\end{array}$ & $38.0(5.3)$ & $35.7(5.9)^{* *}$ & $36.6(6.8)$ & $36.6(6.5)$ \\
\hline \multicolumn{10}{|c|}{$\begin{array}{l}\text { Depression (Beck depression } \\
\text { inventory) mean (SD) }\end{array}$} \\
\hline & Baseline & $\begin{array}{l}\text { End of } \\
\text { treatment }\end{array}$ & 12 months & 72 months & & Baseline & End of treatment & 6 months & 12 months \\
\hline CBT-TL $(n=44)$ & $15.14(9.16)$ & $9.16(7.80)$ & $8.23(11.31)$ & NR & $\begin{array}{l}\text { CBT-TL } \\
(n=45)\end{array}$ & $15.2(6.9)$ & $10.1(8.8)$ & $8.1(7.3)$ & $9.1(7.9)$ \\
\hline BWL-TL $(n=36)$ & $11.82(6.72)$ & $9.19(6.54)$ & $7.76(6.48)$ & NR & $\begin{array}{l}\text { BWL-TL } \\
(n=45)\end{array}$ & $15.9(8.4)$ & $11.1(8.3)$ & $11.1(8.7)$ & $9.6(7.7)$ \\
\hline
\end{tabular}

$* p<.001$.

$*_{*}^{*} p<0.05$; all other comparisons were non-significant.

BWL-TL, behavioural weight loss, therapist-led; CBT-TL, cognitive behavioural therapy, therapist-led; $n$, number of participants; NR, not reported. 
the pharmaceutical findings were moderate for no difference. Three CBT or CBT-BWL comparisons were from trials with mixed or conflicting results and were graded insufficient evidence. Finally, one CBT-BWL comparison was low for CBT benefit; another was moderate for BWL benefit.

\section{Treatment comparisons presented in single randomized controlled trials}

The trials detailed previously were the only ones identified in the search that allowed for combined analysis. However, the search did reveal an additional 23 treatment comparisons, including one pharmacological comparison and 22 psychological comparisons (Appendices $\mathrm{C}-\mathrm{G}$ ). With regard to the former, a single head-to-head trial compared the SGAs fluoxetine and sertraline (Leombruni et al., 2008); although both interventions produced reductions in binge-eating frequency, body weight, eating-related psychopathology, and symptoms of depression, differences between the two treatments were not significant. Given that the results were confined to a single trial in a small sample $(N=44)$, the SOE was insufficient to determine their comparative effectiveness. With regard to the psychological interventions, although the remaining 22 treatment comparisons involved somewhat similar modalities, we judged, based on our protocol and during data synthesis, that the comparators or formats of the interventions (or both) were too dissimilar to allow for any combined analysis (even qualitatively). Because of this decision and because the comparisons were restricted to relatively small trials, we graded the SOE for all outcomes for these comparisons as insufficient.
The treatments compared in these single trials focused on CBT in four trials (Carter \& Fairburn, 1998; Hilbert \& TuschenCaffier, 2004; Le Grange et al., 2002; Ricca et al., 2010); BWL or dietary interventions in four trials (De Zwaan et al., 2005; Grilo \& Masheb, 2005; Grilo et al., 2005; Masheb et al., 2011); dialectical behavioural therapy (DBT) in one trial (Safer et al., 2010); IPT in three trials (Tasca et al., 2006; Wilfley et al., 2002; Wilson et al., 2010); and inpatient treatment programs in three trials (Castelnuovo et al., 2011; Cesa et al., 2013; Riva et al., 2002).

\section{Discussion}

\section{Clinical implications of findings}

Results from the current comparative effectiveness review on interventions for $\mathrm{BED}$ are based on 12 relevant trials of pharmaceutical interventions and 18 of various psychological interventions. Network meta-analysis and qualitative results revealed important information on which interventions might be more effective in improving at least one type of outcome for patients with BED; these treatments include lisdexamfetamine, therapist-led CBT, and BWL. These interventions produced reductions in key BED outcomes including binge-eating abstinence and binge-eating frequency, but they did not demonstrate superiority on all relevant outcomes (e.g., BMI, depressive symptoms). Such information is crucial for health care decisionmaking as patients and clinicians face many options from which to choose; thus, our results help key stakeholders make informed decisions on which treatments might provide important benefits.

Table 3 Strength of evidence for comparative effectiveness of interventions for binge-eating disorder

\begin{tabular}{|c|c|c|c|c|c|}
\hline Outcome & Treatment group 1 & Treatment group 2 & $N$ (Trials) & Summary & Strength of evidence \\
\hline \multirow[t]{5}{*}{ Abstinence from binge eating } & Second-generation antidepressants & Lisdexamphetamine & $791(11)$ & No difference* & Moderate for no difference \\
\hline & CBT Therapist-led & CBT Partially Therapist-led & $158(2)$ & No difference $* *$ & Low for no difference \\
\hline & CBT Therapist-led & CBT Structured Self-help & $158(2)$ & Mixed results- & Insufficient \\
\hline & CBT Partially Therapist-led & CBT Structured Self-help & $164(2)$ & No difference $* *$ & Low for no difference \\
\hline & CBT Therapist-led & BWL Therapist-led & $170(2)$ & Mixed results & Insufficient \\
\hline \multirow[t]{5}{*}{ Binge-eating frequency } & Second-generation antidepressants & Lisdexamphetamine & $649(6)$ & No difference* & Moderate for no difference \\
\hline & CBT Therapist-led & CBT Partially Therapist-led & $158(2)$ & No difference** & Low for no difference \\
\hline & CBT Therapist-led & CBT Structured Self-help & $158(2)$ & Mixed results & Insufficient \\
\hline & CBT Partially Therapist-led & CBT Structured Self-help & $164(2)$ & No difference ${ }^{* *}$ & Low for no difference \\
\hline & CBT Therapist-led & BWL Therapist-led & $170(2)$ & CBT better** & Low for CBT benefit \\
\hline \multirow[t]{4}{*}{ Body mass index } & CBT Therapist-led & CBT Partially Therapist-led & $158(2)$ & No difference** & Low for no difference \\
\hline & CBT Therapist-led & CBT Structured Self-help & $158(2)$ & No difference** & Low for no difference \\
\hline & CBT Partially Therapist-led & CBT Structured Self-help & $164(2)$ & No difference ${ }^{* *}$ & Low for no difference \\
\hline & CBT Therapist-led & BWL Therapist-led & $170(2)$ & BWL better* & Moderate for BWL benefit \\
\hline \multirow[t]{4}{*}{ Symptoms of depression } & CBT Therapist-led & CBT Partially Therapist-led & $158(2)$ & No difference** & Low for no difference \\
\hline & CBT Therapist-led & CBT Structured Self-help & $158(2)$ & No difference** & Low for no difference \\
\hline & CBT Partially Therapist-led & CBT Structured Self-help & $164(2)$ & No difference** & Low for no difference \\
\hline & CBT Therapist-led & BWL Therapist-led & $170(2)$ & No difference ${ }^{* *}$ & Low for no difference \\
\hline Eating-related psychopathology & Second-generation antidepressants & Lisdexamphetamine & $643(6)$ & No difference* & Moderate for no difference \\
\hline
\end{tabular}

BWL, behavioural weight loss; CBT, cognitive behavioural therapy.

*End of treatment

**End of followup. 
For example, comparisons of pharmacological interventions found lisdexamfetamine to be superior to SGAs (as a class) in increasing binge abstinence (moderate SOE for lisdexamfetamine

benefit); however, neither treatment was better than the other for decreasing binge-eating frequency and obsessions and compulsions (moderate SOE for no difference). Similarly, therapist-led CBT was superior to therapist-led BWL in decreasing binge frequency at both end of treatment and up to 12-month followup (low SOE). By contrast, therapist-led BWL was better than CBT in reducing BMI, but only at end of treatment (moderate $\mathrm{SOE}$ ); patients receiving $\mathrm{BWL}$ tended to regain the weight they had lost during treatment, so the benefits from BWL did not persist over time. Comparisons of CBT and BWL on other relevant outcomes (e.g., BMI or depressive symptoms) revealed nonsignificant differences (low SOE for no difference). Additionally, data regarding several levels of therapist involvement in CBT were mixed; the superiority of therapist-led CBT over structured self-help CBT on binge-eating frequency and bingeeating abstinence was found only in the larger of two trials.

The larger pattern of nonsignificant differences between treatments does not imply a lack of significant positive effects on important patient outcomes. Various psychological interventions (e.g., CBT, IPT, and DBT) all improved relevant BED outcomes. However, because both treatment arms produced significant effects over baseline levels, these trials did not reflect superiority for major outcomes of interest in comparisons across the various treatments. Thus, despite substantial evidence supporting the efficacy of many of these interventions (Brownley et al., 2016), the extent to which one treatment is more effective than another has been demonstrated only for lisdexamfetamine (versus SGAs) and therapist-led CBT and BWL (compared with each other) and, then, only for certain outcomes.

That lisdexamfetamine demonstrated superiority to SGAs only on the outcome of binge-eating abstinence merits particular consideration given lisdexamfetamine's recent FDA approval for treating BED patients. Data from the early clinical trials suggested that this medication is a safe and efficacious intervention that helps improve relevant binge-eating outcomes and reduce weight (Brownley et al., 2016). However, not all individuals with BED may be candidates for this intervention, even with its FDA labelling, given that the US Drug Enforcement Administration classifies lisdexamfetamine as a Schedule II drug. This means that clinicians

should not generalize current findings to patients with a history of stimulant or other substance use disorder, suicide attempt, mania, or cardiac disease, as such patients might be more susceptible to abuse or side effects than patients without such a history.

Our network meta-analysis suggests, therefore, that SGAs represent a reasonable pharmacological alternative option for reducing binge eating and improving eating-related psychopathology for patients who are not candidates for lisdexamfetamine. Our meta-analyses used eight different SGAs; each had demonstrated significant reductions in binge eating and improvements in eating-related psychopathology (Berkman et al., 2015). Having alternative pharmacological options increases the ability of clinicians to manage BED effectively across their patient populations. Although a lack of replication for specific SGAs required us to analyse them as a class, the collective evidence is encouraging because this synthesis documents several effective pharmaceutical options from which clinicians and BED patients can choose if they prefer a medication regimen.

Similarly, in relation to psychological interventions, the collective results from this paper and the larger report suggest that patients and clinicians have a variety of effective choices. Not surprisingly, therapist-led CBT appears to be more effective at reducing binge-eating frequency, whereas therapist-led BWL was better at reducing weight. These results might be expected given that CBT for BED was designed to help patients either abstain from or reduce overall binge eating, whereas BWL was designed primarily to aid with weight loss. This pattern of results suggests that it is imperative for patients and clinicians to identify mutual goals for treatment in an effort to select interventions that have the best fit. Additionally, despite the strength of the evidence for therapist-led CBT, we note that many individuals with BED do not have access to therapists who are knowledgeable about BED-specific treatments. Therefore, the generalizability and implementation of the findings from the current review may be limited insofar as eating disorder specialists are not widely available. Our review does highlight favourable outcomes from a self-help approach to CBT (Carter \& Fairburn, 1998; Grilo \& Masheb, 2005; Peterson et al., 2009; Peterson et al., 1998; Wilson et al., 2010). Such a program might help overcome the above barrier to treatment. Moreover, it is consistent with practice guidelines from the National Institute of Health and Care Excellence (NICE) in the United Kingdom, which recommend self-help formats of CBT.

\section{Limitations in the evidence base}

Despite the review revealing 27 treatment comparisons relevant for comparative effectiveness analyses, our ability to draw more definitive conclusions was considerably influenced by the fact that 23 of the 27 relevant treatment comparisons were limited to a single trial (SOE insufficient in all cases). Although results of these individual trials typically reflected improvements in BED outcomes in one or both patient groups, a lack of replication prevented us from synthesizing evidence across studies. For that reason, we could not reach conclusions about the comparative effectiveness of these approaches. Interventions such as IPT and DBT, albeit in single studies, demonstrated promising results at both end of treatment and long-term followup. Hence, these approaches may well merit additional trials to determine their comparative effectiveness.

Furthermore, the applicability of many of our results is constrained as well. For instance, the majority of the trials in this review involved primarily middle-aged white women, many of whom were overweight or obese. Therefore, the extent to which these findings can be extended to other age groups, to men, to more ethnically and racially diverse populations, or to those $\mathrm{BED}$ patients who may be closer to normal weight or BMI is unclear.

Finally, although some of the comparative effectiveness trials of psychological therapies reported data past the end of treatment measurements, none of the pharmacological trials reported data beyond that point. This scarcity of high-quality, longer-term evidence affects our ability to understand how long the shorterterm benefits persist and, subsequently, how long an individual might need to be prescribed a particular medication. 


\section{Implications for future research}

The collective evidence from this comparative effectiveness review is encouraging in that nearly all the interventions studied showed improvements in the anticipated directions for binge-eating outcomes and associated psychopathology. Both pharmacological and psychological interventions were effective at achieving impressive levels of binge abstinence (even if not always 100\%), reducing binge-eating frequency, and improving eating-related psychopathology. Thus, both patients and clinicians have at their disposal a variety of interventions from which to choose for the management of BED. Future research may not be warranted for examining the efficacy of many of these interventions (given competing needs to investigate other aspects of BED treatment). Rather, comparative effectiveness information and studies testing ways to disseminate and implement programs based on our results may be more relevant for setting or clarifying treatment expectations for patients.

Future studies should involve more head-to-head trials of active interventions that have demonstrated initial success in efficacy trials. For example, an RCT comparing a psychological intervention, therapist-led CBT, with lisdexamfetamine pharmacotherapy would allow for a better understanding of which of these robust interventions might prove best for improving BED outcomes, particularly taking patient characteristics adequately into account. Combination trials-that is, those involving both proven medications and proven psychological or behavioural interventions compared with a single intervention-might well shed further light on potentially effective options for care. Trials involving stepped-care models of treatment are also needed. They would allow investigators to determine which interventions are appropriate depending on BED illness severity or duration (or both). Some experts have proposed examining how best to use 'rapid responders' as a way to deliver care more efficiently over time (Grilo, White, Masheb, \& Gueorguieva, 2015; Grilo et al., 2012; Masheb \& Grilo, 2007).

Finally, the trials we could use for this review clearly allow researchers, clinicians, and patients to identify effective interventions. What is missing, however, is widespread dissemination of the results and of the treatments themselves, particularly with respect to the psychological interventions. With regard to these approaches, the absence of a sustainable infrastructure often prevents clinicians and administrators from easily incorporating research findings about psychological interventions into clinical practice. For instance, many of the psychological treatment trials involve doctoral-level therapists, and trials take place outside traditional health care settings or systems that might be able to implement such programs. Thus, the need is critical for researchers to develop innovative ways to translate the findings from such trials into clinical practice such that all individuals with BED, not just those who are able to access clinical trials, might benefit from evidence-based treatment.

\section{Disclaimer}

The authors of this manuscript are responsible for its content. Statements in the manuscript should not be construed as endorsement by the Agency for Healthcare Research and Quality or the U.S. Department of Health and Human Services. The Agency for Healthcare Research and Quality retains a licence to display, reproduce, and distribute the data and the report from which this manuscript was derived under the terms of the agency's contract with RTI International.

\section{Acknowledgements}

We thank both Lauren Breithaupt and Margaret Sala for their assistance with abstract reviews. We are grateful to Isabelle Lanser, Michela Quaranta, Loraine Monroe, Laura Morgan, and Morgan Walker for their assistance with table development and manuscript preparation. We are indebted to Meera Viswanathan, $\mathrm{PhD}$, Ina F. Wallace, PhD, and Lynn Whitener, DrPH, MSLS, for their assistance with the full systematic review from which this manuscript was derived. All authors received support from Contract no. 290-2012-00008-I. Dr. Bulik is a grant recipient from Shire Pharmaceuticals, has consulted for Ironshore, and acknowledges funding from the Swedish Research Council (VR Dnr: 538-2013-8864). Dr. Peat acknowledges funding from Sunovion Pharmaceuticals and Shire Pharmaceuticals and has consulted for LEK Consulting, Nexus Global Solutions, and Trinity Partners. Both investigators recused themselves from all discussions of any trials supported by these funders. No other investigators have financial disclosures to report. This project was funded under Contract no. 290-2012-00008-I from the Agency for Healthcare Research and Quality, U.S. Department of Health and Human Services.

\section{REFERENCES}

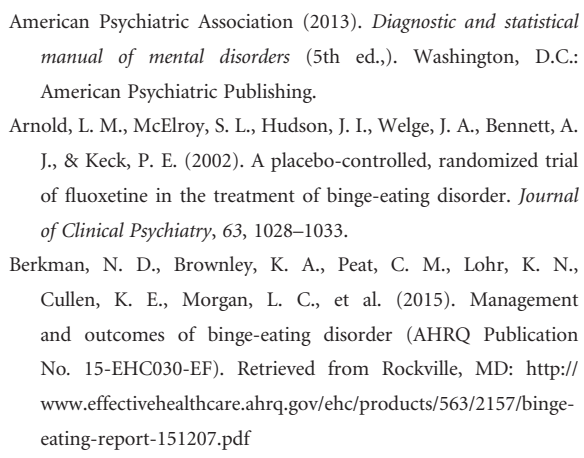

Berkman, N. D., Bulik, C. M., Brownley, K. A., Lohr, K. N., Sedway, J. A., Rooks, A., \& Gartlehner, G. (2006). Management of eating disorders. Evidence Report Technology Assessment (Full Rep) (135), 1-166.

Berkman, N. D., Lohr, K. N., Ansari, M., McDonagh, M., Balk, E., Whitlock, E., et al. (2013). Grading the strength of a body of evidence when assessing health care interventions for the effective health care program of the Agency for Healthcare Research and Quality: an update (AHRQ Publication No. 13 (14)-EHC130-EF). Retrieved from Rockville, MD: http://www. effectivehealthcare.ahrq.gov/reports/final.cfm

Berkman, N. D., Lohr, K. N., Ansari, M., McDonagh, M., Balk, E., Whitlock, E., et al. (2014). AHRQ methods for effective health care. Grading the strength of a body of evidence when assessing health care interventions for the effective health care program of the Agency for Healthcare Research and Quality: An update; 2008. Methods guide for effectiveness and comparative effectiveness reviews. AHRQ Publication No. 10(14)-EHC063EF. Rockville, MD: Agency for Healthcare Research and Quality. Berkman, N. D., Lohr, K. N., \& Bulik, C. M. (2007). Outcomes of eating disorders: A systematic review of the literature. International Journal of Eating Disorders, 40, 293-309. Brownell, K. (2000). The LEARN program for weight management. Dallas, TX: American Health Publishing Company.

Brownley, K. A., Berkman, N. D., Peat, C. M., Lohr, K. N., Cullen, K. E., Bann, C. M., et al. (2016). Binge-eating disorder in adults: A systematic review and meta-analysis. Annals of Internal Medicine, 165, 409-420. 
Brownley, K. A., Berkman, N. D., Sedway, J. A., Lohr, K. N., \& Bulik, C. M. (2007). Binge eating disorder treatment: a systematic review of randomized controlled trials. International Journal of Eating Disorders, 40, 337-348.

Bruce, B., \& Wilfley, D. (1996). Binge eating among the overweight population: A serious and prevalent problem. Journal of the American Dietetic Association, 96, 58-61.

Carter, J. C., \& Fairburn, C. G. (1998). Cognitive-behavioral selfhelp for binge eating disorder: A controlled effectiveness study. Journal of Consulting and Clinical Psychology, 66, 616-623.

Castelnuovo, G., Manzoni, G. M., Villa, V., Cesa, G. L., \& Molinari, E. (2011). Brief strategic therapy vs cognitive behavioral therapy for the inpatient and telephone-based outpatient treatment of binge eating disorder: The STRATOB randomized controlled clinical trial. Clinical Practice and Epidemiology in Mental Health, 7, 29-37.

Cesa, G. L., Manzoni, G. M., Bacchetta, M., Castelnuovo, G., Conti, S., Gaggioli, A., et al. (2013). Virtual reality for enhancing the cognitive behavioral treatment of obesity with binge eating disorder: Randomized controlled study with one-year followup. Journal of Medical Internet Research, 15, el13.

Chatoor, I., \& Krupnick, J. (2001). The role of non-specific factors in treatment outcome of psychotherapy studies. European Child and Adolescent Psychiatry, 10, I19-I25.

De Zwaan, M., Mitchell, J. E., Crosby, R. D., Mussell, M. P., Raymond, N. C., Specker, S. M., et al. (2005). Short-term cognitive behavioral treatment does not improve outcome of a comprehensive very-low-calorie diet program in obese women with binge eating disorder. Behavior Therapy, 36, 89-99.

Deal, L. S., Wirth, R. J., Gasior, M., Herman, B. K., \& McElroy, S. L. (2015). Validation of the yale-brown obsessive compulsive scale modified for binge eating. International Journal of Eating Disorders, 48, 994-1004.

Fairburn, C. G. (1995). Overcoming binge eating: The proven program to learn why you binge and how you can stop. New York, NY: Guilford.

Fairburn, C. G., Marcus, M. D., \& Wilson, G. T. (1993). Cognitive behaviour therapy for binge eating and bulimia nervosa: a comprehensive treatment manual. In C. G. Fairburn, \& G. T. Wilson (Eds.), Binge eating: Nature, assessment, and treatment (, pp. 361-404). New York: Guilford Press.

Glenny, A. M., Altman, D. G., Song, F., Sakarovitch, C., Deeks, J. J., D'Amico, R., et al. (2005). Indirect comparisons of competing interventions. Health Technology Assessment, 9, 1-134 iii-iv.

Grilo, C. M., \& Masheb, R. M. (2005). A randomized controlled comparison of guided self-help cognitive behavioral therapy and behavioral weight loss for binge eating disorder. Behaviour Research and Therapy, 43, 1509-1525.

Grilo, C. M., Masheb, R. M., \& Wilson, G. T. (2005). Efficacy of cognitive behavioral therapy and fluoxetine for the treatment of binge eating disorder: A randomized double-blind placebocontrolled comparison. Biological Psychiatry, 57, 301-309.

Grilo, C. M., Masheb, R. M., Wilson, G. T., Gueorguieva, R., \& White, M. A. (2011). Cognitive-behavioral therapy, behavioral weight loss, and sequential treatment for obese patients with binge-eating disorder: A randomized controlled trial. Journal of Consulting and Clinical Psychology, 79, 675-685.

Grilo, C. M., White, M. A., Masheb, R. M., \& Gueorguieva, R. (2015). Predicting meaningful outcomes to medication and self-help treatments for binge-eating disorder in primary care: The significance of early rapid response. Journal of Consulting and Clinical Psychology, 83, 387-394.
Grilo, C. M., White, M. A., Wilson, G. T., Gueorguieva, R., \& Masheb, R. M. (2012). Rapid response predicts 12-month posttreatment outcomes in binge-eating disorder: Theoretical and clinical implications. Psychological Medicine, 42, 807-817.

Grucza, R. A., Przybeck, T. R., \& Cloninger, C. R. (2007). Prevalence and correlates of binge eating disorder in a community sample. Comprehensive Psychiatry, 48, 124-131.

Guerdjikova, A. I., McElroy, S. L., Kotwal, R., Welge, J. A., Nelson, E., Lake, K., et al. (2008). High-dose escitalopram in the treatment of binge-eating disorder with obesity: A placebocontrolled monotherapy trial. Human Psychopharmacology, 23, $1-11$.

Guerdjikova, A. I., McElroy, S. L., Winstanley, E. L., Nelson, E. B., Mori, N., McCoy, J., et al. (2012). Duloxetine in the treatment of binge eating disorder with depressive disorders: A placebocontrolled trial. International Journal of Eating Disorders, 45, 281-289.

Gupta, U., \& Verma, M. (2013). Placebo in clinical trials. Perspectives in Clinical Research, 4, 49-52.

Higgins, J. P., Altman, D. G., Gotzsche, P. C., Juni, P., Moher, D., Oxman, A. D., et al. (2011). The Cochrane Collaboration's tool for assessing risk of bias in randomised trials. BMJ, 343, d5928.

Hilbert, A., Bishop, M. E., Stein, R. I., Tanofsky-Kraff, M., Swenson, A. K., Welch, R. R., et al. (2012). Long-term efficacy of psychological treatments for binge eating disorder. British Journal of Psychiatry, 200, 232-237.

Hilbert, A., Hildebrandt, T., Agras, W. S., Wilfley, D. E., \& Wilson, G. T. (2015). Rapid response in psychological treatments for binge eating disorder. Journal of Consulting and Clinical Psychology, 83, 649-654.

Hilbert, A., \& Tuschen-Caffier, B. (2004). Body image interventions in cognitive-behavioural therapy of binge-eating disorder: A component analysis. Behaviour Research and Therapy, 42, 1325-1339.

Hudson, J. I., Hiripi, E., Pope, H. G. Jr., \& Kessler, R. C. (2007). The prevalence and correlates of eating disorders in the National Comorbidity Survey Replication. Biological Psychiatry, 61, 348-358.

Hudson, J. I., McElroy, S. L., Raymond, N. C., Crow, S., Keck, P. E. Jr., Carter, W. P., et al. (1998). Fluvoxamine in the treatment of binge-eating disorder: A multicenter placebocontrolled, double-blind trial. American Journal of Psychiatry, 155, 1756-1762.

Kessler, R. C., Berglund, P. A., Chiu, W. T., Deitz, A. C., Hudson, J. I., Shahly, V., et al. (2013). The prevalence and correlates of binge eating disorder in the World Health Organization World Mental Health Surveys. Biological Psychiatry, 73, 904-914.

Le Grange, D., Gorin, A., Dymek, M., \& Stone, A. (2002). Does ecological momentary assessment improve cognitive behavioural therapy for binge eating disorder? A pilot study. European Eating Disorders Review, 10, 316-328.

Leombruni, P., Pierò, A., Lavagnino, L., Brustolin, A., Campisi, S., \& Fassino, S. (2008). A randomized, double-blind trial comparing sertraline and fluoxetine 6-month treatment in obese patients with binge eating disorder. Progress in Neuropsychopharmacology and Biological Psychiatry, 32, 1599-1605.

Marek, R. J., Ben-Porath, Y. S., Ashton, K., \& Heinberg, L. J. (2014). Impact of using DSM-5 criteria for diagnosing binge eating disorder in bariatric surgery candidates: Change in prevalence rate, demographic characteristics, and scores on the minnesota multiphasic personality inventory - 2 restructured form (MMPI2-RF). International Journal of Eating Disorders, 47, 553-557.
Margraf, J. (2000). Aus dick wird nicht du"nn. Falsche Erwartungshaltungen. In E. Roche (Ed.), Hu"lle und Fu“lle. Dem Fett auf den Leib geru" ckt. Basel: Hoffmann-La Roche.

Masheb, R. M., \& Grilo, C. M. (2007). Rapid response predicts treatment outcomes in binge eating disorder: Implications for stepped care. Journal of Consulting and Clinical Psychology, 75, 639-644.

Masheb, R. M., Grilo, C. M., \& Rolls, B. J. (2011). A randomized controlled trial for obesity and binge eating disorder: Lowenergy-density dietary counseling and cognitive-behavioral therapy. Behaviour Research and Therapy, 49, 821-829.

McElroy, S. L., Casuto, L. S., Nelson, E. B., Lake, K. A., Soutullo, C. A., Keck, P. E. Jr., et al. (2000). Placebo-controlled trial of sertraline in the treatment of binge eating disorder. American Journal of Psychiatry, 157, 1004-1006.

McElroy, S. L., Hudson, J., Ferreira-Cornwell, M. C., Radewonuk, J., Whitaker, T., \& Gasior, M. (2015a). Lisdexamfetamine dimesylate for adults with moderate to severe binge eating disorder: Results of two pivotal phase 3 randomized controlled trials. Neuropsychopharmacology doi:https://doi.org/10.1038/ npp. 2015.275

McElroy, S. L., Hudson, J. I., Malhotra, S., Welge, J. A., Nelson, E. B., \& Keck, P. E. Jr. (2003). Citalopram in the treatment of bingeeating disorder: A placebo-controlled trial. Journal of Clinical Psychiatry, 64, 807-813.

McElroy, S. L., Hudson, J. I., Mitchell, J. E., Wilfley, D., FerreiraCornwell, M. C., Gao, J., et al. (2015b). Efficacy and safety of lisdexamfetamine for treatment of adults with moderate to severe binge-eating disorder: A randomized clinical trial. JAMA Psychiatry, 72, 235-246.

Munsch, S., Biedert, E., Meyer, A., Michael, T., Schlup, B., Tuch, A., et al. (2007). A randomized comparison of cognitive behavioral therapy and behavioral weight loss treatment for overweight individuals with binge eating disorder. International Journal of Eating Disorders, 40, 102-113.

Munsch, S., Meyer, A. H., \& Biedert, E. (2012). Efficacy and predictors of long-term treatment success for cognitivebehavioral treatment and behavioral weight-loss-treatment in overweight individuals with binge eating disorder. Behaviour Research and Therapy, 50, 775-785.

Neupane, B., Richer, D., Bonner, A. J., Kibret, T., \& Beyene, J. (2014). Network meta-analysis using R: A review of currently available automated packages. PloS One, 9, el15065.

Peat, C. M., Brownley, K. A., Berkman, N. D., \& Bulik, C. M. (2012). Binge eating disorder: Evidence-based treatments. Current Psychology, 11, 32-39.

Peterson, C. B., Mitchell, J. E., Crow, S. J., Crosby, R. D., \& Wonderlich, S. A. (2009). The efficacy of self-help group treatment and therapist-led group treatment for binge eating disorder. American Journal of Psychiatry, 166, 1347-1354.

Peterson, C. B., Mitchell, J. E., Engbloom, S., Nugent, S., Mussell, M. P., Crow, S. J., et al. (2001). Self-help versus therapist-led group cognitive-behavioral treatment of binge eating disorder at followup. International Journal of Eating Disorders, 30, 363-374.

Peterson, C. B., Mitchell, J. E., Engbloom, S., Nugent, S., Mussell, M. P., \& Miller, J. P. (1998). Group cognitive-behavioral treatment of binge eating disorder: A comparison of therapist-led versus self-help formats. International Journal of Eating Disorders, 24, 125-136.

Pisetsky, E. M., Durkin, N. E., Crosby, R. D., Berg, K. C., Mitchell, J. E., Crow, S. J., et al. (2015). Examination of early group dynamics and treatment outcome in a randomized controlled trial of group cognitive behavior therapy for binge eating disorder. Behaviour Research and Therapy, 73, 74-78. 
Ricca, V., Castellini, G., Mannucci, E., Lo Sauro, C., Ravaldi, C., Rotella, C. M., et al. (2010). Comparison of individual and group cognitive behavioral therapy for binge eating disorder. A randomized, three-year follow-up study. Appetite, 55, 656-665.

Riva, G., Bacchetta, M., Baruffi, M., \& Molinari, E. (2002). Virtualreality-based multidimensional therapy for the treatment of body image disturbances in binge eating disorders: A preliminary controlled study. IEEE Transactions on Information Technology in Biomedicine, 6, 224-234.

Robinson, A. H., \& Safer, D. L. (2012). Moderators of dialectical behavior therapy for binge eating disorder: Results from a randomized controlled trial. International Journal of Eating Disorders, 45, 597-602.

Rücker, G. (2012). Network meta-analysis, electrical networks and graph theory. Research Synthesis Methods, 3, 312-324.

Safer, D. L., \& Joyce, E. E. (2011). Does rapid response to two group psychotherapies for binge eating disorder predict abstinence? Behaviour Research and Therapy, 49, 339-345.

Safer, D. L., Robinson, A. H., \& Jo, B. (2010). Outcome from a randomized controlled trial of group therapy for binge eating disorder: comparing dialectical behavior therapy adapted for binge eating to an active comparison group therapy. Behavior Therapy, 41, 106-120.

Shire Development LLC. (2014a). SPD489 in adults aged $18-55$ years with moderate to severe binge eating disorder. ClinicalTrials.gov. Identifier: NCT01718483. Retrieved from https://www. clinicaltrials.gov/ct2/show/results?term=vyvanse+binge-eating + disorder\&rank $=3$
Shire Development LLC. (2014b). SPD489 in adults aged 18-55 years with moderate to severe binge eating disorder. ClinicalTrials.gov. Identifier: NCT01718509. Retrieved from https://www. clinicaltrials.gov/ct2/show/results?term=vyvanse+binge-eating + disorder\&rank $=4 \&$ sect $=$ X301256

Spitzer, R. L., Stunkard, A., Yanovski, S., Marcus, M. D., Wadden, T., Weng, R., et al. (1993a). Binge eating disorder should be included in DSM-IV: a reply to Fairburn et al.'s "the classification of recurrent overeating: the binge eating disorder proposal.". International Journal of Eating Disorders, 13, 161-169.

Spitzer, R. L., Yanovski, S., Wadden, T., Wing, R., Marcus, M. D., Stunkard, A., et al. (1993b). Binge eating disorders: its further validation in a multisite study. International Journal of Eating Disorders, 13, 137-153.

Sysko, R., Hildebrandt, T., Wilson, G. T., Wilfley, D. E., \& Agras, W. S. (2010). Heterogeneity moderates treatment response among patients with binge eating disorder. Journal of Consulting and Clinical Psychology, 78, 681-690.

Tasca, G., Ritchie, K., Conrad, G., Balfour, L., Gayton, J., Lybanon, V., et al. (2006). Attachment scales predict outcome in a randomized controlled trial of two group therapies for binge eating disorder: An aptitude by treatment interaction. Psychotherapy Research, 16, 106-121.

Tasca, G. A., Balfour, L., Presniak, M. D., \& Bissada, H. (2012). Outcomes of specific interpersonal problems for binge eating disorder: comparing group psychodynamic interpersonal psychotherapy and group cognitive behavioral therapy. International Journal of Group Psychotherapy, 62, 197-218.

Trace, S. E., Thornton, L. M., Root, T. L., Mazzeo, S. E., Lichtenstein, P., Pedersen, N. L., et al. (2012). Effects of reducing the frequency and duration criteria for binge eating on lifetime prevalence of bulimia nervosa and binge eating disorder: implications for DSM-5. International Journal of Eating Disorders, 45, 531-536.

U.S. Food and Drug Administration. (2015). FDA news release. FDA expands uses of Vyvanse to treat binge-eating disorder. Retrieved from http://www.fda.gov/newsevents

van Valkenhoef, G., Lu, G., de Brock, B., Hillege, H., Ades, A. E., \& Welton, N. J. (2012). Automating network meta-analysis. Research Synthesis Methods, 3, 285-299.

van Valkenhoef, G., Kuiper, J. Network meta-analysis using Bayesian methods. 2016.

Whisman, M. A., Dementyeva, A., Baucom, D. H., \& Bulik, C. M. (2012). Marital functioning and binge eating disorder in married women. International Journal of Eating Disorders, 45, 385-389.

White, M. A., \& Grilo, C. M. (2013). Bupropion for overweight women with binge-eating disorder: A randomized, doubleblind, placebo-controlled trial. Journal of Clinical Psychiatry, 74, 400-406.

Wilfley, D. E., Welch, R. R., Stein, R. I., Spurrell, E. B., Cohen, L. R., Saelens, B. E., et al. (2002). A randomized comparison of group cognitive-behavioral therapy and group interpersonal psychotherapy for the treatment of overweight individuals with binge-eating disorder. Archives of General Psychiatry, 59, 713-721.

Wilson, G. T., Wilfley, D. E., Agras, W. S., \& Bryson, S. W. (2010). Psychological treatments of binge eating disorder. Archives of General Psychiatry, 67, 94-101.

\section{Supporting information}

Additional Supporting Information may be found online in the supporting information tab for this article. 